\title{
Improving doctors' prescribing behaviour through reflection on guidelines and prescription feedback: a randomised controlled study
}

\author{
Per Lagerløv, Mitchell Loeb, Marit Andrew, Per Hjortdahl
}

\begin{abstract}
Background-It is difficult to put research findings into clinical practice by either guidelines or prescription feedback.

Aim-To study the effect on the quality of prescribing by a combined intervention of providing individual feedback and deriving quality criteria using guideline recommendations in peer review groups.

Methods-199 general practitioners in 32 groups were randomised to participate in peer review meetings related to either asthma or urinary tract infections. The dispensing by the participating doctors of antiasthmatic drugs and antibiotics during the year before the intervention period provided the basis for prescription feedback. The intervention feedback was designed to describe the treatment given in relation to recommendations in the national guidelines. In each group the doctors agreed on quality criteria for their own treatment of the corresponding diseases based on these recommendations. Comparison of their prescription feedback with their own quality criteria gave each doctor the proportion of acceptable and unacceptable treatments.

Main outcome measure-Difference in the prescribing behaviour between the year before and the year after the intervention.
\end{abstract}

Department of

Pharmacotherapeutics,

University of Oslo,

Oslo, Norway

P Lagerløv, research fellow

SINTEF Unimed, Oslo, Norway

M Loeb, research scientist

Norwegian Board of

Health, Oslo, Norway

M Andrew, deputy director general

Department of General Practice, University of Oslo, Oslo, Norway

P Hjortdahl, professor

Correspondence to: Dr P Lagerløv, Department of Pharmacotherapeutics,

University of Oslo, P O Box

1065 Blindern, N-0316 Oslo,

Norway

per.lagerlov@netcom.no

Accepted 13 July 2000
Presented with feedback on their own prescribing, they learned what they did right and wrong. This provided a foundation for improvement and the process thus instigated resulted in the doctors providing better quality patient care. (Quality in Health Care 2000;9:159-165)

Keywords: quality assessment; quality improvement; prescription feedback; continuing medical education; asthma; urinary tract infection

It is difficult to put research findings into clinical practice. ${ }^{12}$ Providing doctors with knowledge on how to treat diseases in accordance with research evidence and guideline recommendations seldom changes the way doctors prescribe drugs. ${ }^{3}$ Multiple approaches applied in combination appear to work best, ${ }^{4}$ sometimes showing a dose-response curve in improving knowledge. ${ }^{5}$ Improved knowledge does not, however, necessarily lead to a corresponding change in behaviour. In behavioural psychology changes in attitudes are frequently perceived to follow rather than precede behaviour modifications. ${ }^{6}$ Feedback on prescribing, giving information on aggregated data, may thus not be sufficient to change behaviour. $^{78}$

In the present study feedback to individual doctors on how they treated their patients was structured in such a way as to make possible a comparison with guideline recommendations. The recommendations needed to be reformulated to enable a quality assessment of patient treatment to be judged from prescription feedback. Such quality criteria were developed during group discussions between doctors participating in the study. Doctors may, during this active process, discover the consequences of new knowledge in relation to their own behaviour. Problem based learning, also known as "problem first learning", 9 places the emphasis on the learner's own initiative to discover problems and how to improve. By discussion in peer review groups the individual doctor's selfefficacy, defined as one's ability to organise and execute a course of action required to produce given results, is substantially increased. ${ }^{10}$ The ability to change clinical practice is influenced by many factors, however. ${ }^{11}$ Problem based learning has found its way into the theory of medical education. ${ }^{12}$

The aim of this study was to apply this theory of medical education to the improvement of quality of patient care. The study examined whether this process improved the quality of treatment among patients with the 
model diseases, asthma and urinary tract infection (UTI), and assessed how well the participating doctors approved of the group process. It further examined whether recorded characteristics of the doctors, their practice, and their change in management routines influenced the improvement.

\section{Methods}

RECRUITMENT AND ESTABLISHMENT OF GROUPS Six hundred and thirty five general practitioners (GPs) from south-east Norway, registered by The Norwegian Medical Association, were invited to participate in the study; 199 (31\%) agreed to take part and were organised into 32 groups with 4-8 GPs in each group. The GPs in each group were all located in a defined geographical area served by 1-3 pharmacies. Groups were block randomised to focus either on asthma or UTI. The randomisation was stratified into three equal sized geographical areas to secure an even distribution of the 16 asthma groups and 16 UTI groups, the former serving as controls for the latter and vice versa. The study is the Norwegian part of the Drug Education Project (DEP), comparing the effect of educational intervention on drug prescribing in five European countries. ${ }^{13}$

DATA FOR FEEDBACK

Asthma was chosen as one of the disease models as graded individual treatment recommendations were available as part of national ${ }^{14}$ and internationa $1^{15}$ guidelines. The overall recommendations state that inhaled $\beta$ agonists should be used when needed, and inhaled steroids should be used regularly to prevent episodes of asthma. No asthma patients should be using inhaled $\beta$ agonists frequently without also using inhaled steroids. Uncomplicated UTI was selected as the other disease model. For this infection short treatment duration is recommended, both nationally ${ }^{14}$ and internationally. ${ }^{16} \mathrm{~A}$ new edition of the national guidelines used in this study are mailed to all Norwegian doctors every second year free of charge.

The GPs agreed to let us record the anti-asthmatic treatments and the relevant drugs for treating UTI dispensed to their patients. This was done by evaluating all their prescriptions at their local pharmacies during the one year period before and the one year period after the intervention. Inhaled $\beta$ agonists and inhaled steroids dispensed to patients

Table 1 The defined daily dose (DDD) of the recorded drugs

\begin{tabular}{ll}
\hline & Defined daily dose $(m g)$ \\
\hline Anti-asthmatics & \\
Inhaled short acting $\beta$ agonists & 0.8 \\
Salbutamol & 2.0 \\
Terbutaline & 0.6 \\
Fenoterol & \\
Inhaled steroids & 0.8 \\
Beclomethasone & 0.8 \\
Budesonide & 1.0 \\
Flunisolide & 0.6 \\
Fluticasone & \\
Anti-infectives & 400 \\
Trimethoprim & 600 \\
Pivmecillinam & 1600 and 320 \\
Sulfamethoxazole and trimethoprim (co-trimoxazole) & \\
\hline
\end{tabular}

aged 17-50 years were recorded and these prescriptions were thus used as an indicator or proxy for asthmatic patients. The age limits were set in order to exclude children where the guideline recommendations deviate from those established for adults, and also to reduce the number of elderly patients included with chronic obstructive pulmonary disease. The amount of each drug dispensed per prescription was recorded in defined daily doses (DDDs), ${ }^{17}$ further described in table 1 . The mean daily dose of inhaled $\beta$ agonists and inhaled steroids dispensed to each patient during one year was calculated.

Trimethoprim, pivmecillinam, and cotrimoxazole were selected as indicators among the different antibiotics used for treating UTI. These are the main drugs for treating uncomplicated UTI in Norway today and they can be dispensed in different sized packages. Broad spectrum penicillins are primarily prescribed for other types of infection or for treating UTI in pregnancy. Only treatments dispensed for a two week course or less and to patients 16 years or older were included in our study. Children and patients with prophylactic long term treatments were excluded. The duration of treatment for each patient was calculated by dividing the total amount dispensed by one DDD. For trimethoprim $75 \%$ of the DDD was used as the normal daily dose as this corresponds to the most frequently prescribed daily dose in Norway.

\section{INTERVENTION}

Each group participated in two evening meetings about one week apart during autumn 1995. The meetings lasted on average 2 hours and 45 minutes. At the first meeting the GPs discussed in groups how they diagnose the illness, and the underlying reasons they find important when deciding on treatment. At the second meeting international and national guidelines for treating asthma or UTI were presented to the respective groups. After plenary discussion the group agreed on common quality criteria for what they found to be acceptable and unacceptable prescribing, based on the guideline recommendations. These criteria were subsequently compared with the prescribing histories of the group as a whole over the previous year, and then the same was done individually for each GP. The way in which these quality improvement tools were applied to asthma and UTI within each group are summarised in boxes 1 and 2, respectively. One of the two project coordinators and a pharmacist from the local pharmacies were present at each meeting. The meetings were conducted according to a structured plan, with the main emphasis being on group discussions and without formal lecturing.

PROCESS EVALUATION OF THE GROUP INTERVENTION

To get an indication of how the groups functioned, the participants anonymously evaluated the group process at the end of the last meeting. Their responses were given on a 
- A $4 \times 4$ table of mean daily dosage intervals of the combined use of inhaled $\beta$ agonists and inhaled steroids was presented to the GPs in each peer review group.

- Without any data in the table the GPs discussed, based on national guideline recommendations, within which of the combined mean daily dosage intervals they would or would not accept the treatment given to a patient.

- They then coloured the mean daily dosage intervals green or red corresponding to their consensus on acceptable or unacceptable treatments. At least $80 \%$ of the groups were able to label consistently 11 of the 16 dosage intervals. The dosage intervals which could not be labelled were coloured yellow.

- All the relevant patients were then ascribed to a mean daily dosage interval in the table according to the relationship between the calculated mean daily dose of inhaled $\beta$ agonist and inhaled steroids dispensed during a year. The table containing data was then presented to the GPs.

- The number of patients in green (acceptable) and red (unacceptable) mean daily dosage intervals represented the prescribing feedback on the quality of treatment. The group of doctors and the individual doctor within each group could thus see what they did right and wrong.

- Examples of acceptable treatments: Acceptably treated patients with less severe asthma received a mean daily dose of inhaled $\beta$ agonists equivalent to less than 1-2 inhalations a day in combination with inhaled steroids equal to less than $400 \mu \mathrm{g} /$ day beclomethasone. Patients with more severe asthma treated acceptably received a mean daily dose of inhaled $\beta$ agonists equivalent to less than $2-4$ inhalations a day in combination with inhaled steroids equal to $400-800 \mu \mathrm{g} /$ day beclomethasone.

- Example of unacceptable treatments: Unacceptably treated patients received a mean daily dose of inhaled $\beta$ agonists equivalent to 1-2 inhalations a day or more without any inhaled steroids.

Box 1 Deciding on treatment quality of asthma.

six point scale with a high score reflecting a good evaluation. They were also asked if they intended to change their management of asthma or UTI as a result of the intervention, and whether they considered this method of educational intervention suitable for improving treatment of other illnesses.

MAIN OUTCOME VARIABLES

The difference in proportions of acceptably and unacceptably treated asthma patients before and after the intervention was calculated within the asthma and control (UTI) groups. The effect measurement of the intervention on
- A table dividing the treatments of UTI into short (4 days or less), medium (more than 4 but less than 7 days), and long (7 to 14 days) ranges of duration was presented to the GPs in peer review groups.

- Without any data in the table the GPs discussed, based on national guideline recommendations, which range of duration they considered acceptable or unacceptable when treating uncomplicated UTI.

- The GPs coloured the ranges of duration green or red corresponding to their consensus on acceptable or unacceptable treatment duration. At least $80 \%$ of the groups were able to label consistently two of the three ranges of duration. The range without a consistent label was coloured yellow.

- The treatments of UTI were then tabulated into each range of duration according to the calculated duration of treatments. The number of treatments within green and red ranges of duration constituted the prescribing feedback. The group of doctors and the individual doctors in each group could thus see what they did right and wrong.

- Acceptable treatment duration of uncomplicated UTI was judged to be 4 days or less.

- Unacceptable treatment duration of uncomplicated UTI was judged to be 7 days or longer.

Box 2 Deciding on treatment quality of uncomplicated urinary tract infection (UTI).

asthma was then calculated as the relative change in these proportions in the asthma groups compared with the control (UTI) groups.

The difference in proportions of short and long treatments for UTI before and after the intervention was calculated within the UTI and control (asthma) groups. The effect measurement of the intervention on UTI was correspondingly the relative change in these proportions in the UTI groups compared with the control (asthma) groups.

CHARACTERISTICS AFFECTING THE DOCTORS' ABILITY TO CHANGE PRESCRIBING BEHAVIOUR Relevant data were collected using questionnaires mailed to the participating GPs, using two reminders when necessary. The GP identification number used in the prescription database identified each questionnaire, enabling linkage of data. The aim was to study whether variations in the change of prescribing behaviour could be explained in part by variations in these factors.

Characteristics of the doctors and their practice In the return letter stating whether or not they wanted to participate in the study the GPs indicated their age (in 1994), sex, number of workdays per week, number of GPs working 
together, and if they were board certified as specialists.

In a questionnaire mailed to the GPs before randomisation into the study groups they specified how long in years they had worked half time or more in general practice, whether they used computers or not, and whether the practice was located in a rural district, a town (up to 30000 inhabitants), or a city. They further specified the number of hours spent on continuing medical education (CME) during the last year, and whether they had taken part in CME activities related to asthma or UTI during the last two years.

\section{Fob satisfaction}

The Norwegian Medical Association undertook in 1993 an extensive survey of Norwegian doctors' self-reported health related to complaints and job stress. ${ }^{18}$ From this study questions relevant to judging the GPs' perceived autonomy, stress, feedback from colleagues, patients and collaborators at work, and their working conditions were selected. These questions were all part of the prerandomisation questionnaire.

Change of routines in management of the diseases These questionnaires were mailed six months after the interventions. All the GPs were asked to respond to each of the following six questions: "For asthma, were there any changes in patient monitoring, in prescription renewals, in giving of information and education to the patients? For UTI, were there any changes in the routines of urine sample collection and analysis, in ways of prescribing, in patient follow up routines?" The GPs were furthermore asked to comment on their routines to ensure a correct interpretation of the question.

\section{STATISTICAL ANALYSIS}

In order to reflect randomisation by group, a multilevel model ${ }^{19}{ }^{20}$ was used which examined the change in prescribing behaviour between the asthma and UTI groups at both the group and GP level. The variance at the group level contributed less than $10 \%$ of the total variance with this model, however. At the GP level linear regression was used to determine whether variations in the change in prescribing behaviour could be explained by variations in the recorded factors describing the GPs and their practice. The computer programs $\mathrm{MlwiN}^{21}$ and SPSS $^{22}$ were employed. Associations in the data were considered to be statistically significant if $\mathrm{p}<0.05$.

Table 2 Characteristics of participating and non-participating general practitioners (GPs)

\begin{tabular}{lllll}
\hline & Mean (SD) & $\begin{array}{l}\text { Percentage } \\
\text { of women }\end{array}$ & $\begin{array}{l}\text { Mean (SD) } \\
\text { number of GPs } \\
\text { working together }\end{array}$ & $\begin{array}{l}\text { Percentages of } \\
\text { GPs board } \\
\text { certified as } \\
\text { specialists }\end{array}$ \\
\hline Participating GPs (n=1994) & $44.1(7.4)$ & 22.6 & $2.4(1.3)$ & 66.8 \\
Non-participating GPs & $\begin{array}{l}45.3(10.0) \\
\mathrm{n}=432^{\star}\end{array}$ & $\begin{array}{ll}21.6 \\
\mathrm{n}=435^{\star}\end{array}$ & $\begin{array}{l}2.5(1.5) \\
\mathrm{n}=182^{\star \star}\end{array}$ & 39.2 \\
& $\mathrm{n}=434^{\star}$ \\
\hline
\end{tabular}

$\star$ Data from records of doctors in Norway.

${ }^{\star}$ Data given in the return letters from GPs invited to participate in the study.

\section{Results}

The sex, mean age, mean number of GPs working together, and proportions of GPs who were board certified as specialists among the participating and non-participating GPs are presented in table 2. Data from the nonparticipating GPs were derived from information given in the return letter and from public sources. ${ }^{23}$

CHANGES IN PRESCRIBING BEHAVIOUR ASCRIBED TO THE INTERVENTION

Asthma treatment

The GPs in the asthma groups increased their proportion of acceptably treated asthma patients relative to the GPs in the control (UTI) groups. The proportion of unacceptably treated asthma patients did not change. The relative increase in the proportion of acceptably treated asthma patients was $5.9 \%$ (variance 2.5), $\mathrm{p}=0.018$, and the relative change in proportion of unacceptably treated asthma patients was $-1.4 \%(2.0), \mathrm{p}=0.48$, using the hierarchical model. The variance at the group level constituted $4.2 \%$ and $5.6 \%$ of the total variance, respectively.

\section{UTI treatment}

The GPs in the UTI groups increased their proportion of short acceptable UTI treatments and reduced their proportion of long unacceptable UTI treatments relative to the GPs in the control (asthma) groups. The relative increase in the proportion of short UTI treatments was $13.1 \%$ (variance 2.3 ), $\mathrm{p}<0.0001$, and the relative decrease in the proportion of long UTI treatments was $-9.6 \%$ (2.7), $\mathrm{p}=0.0004$, using the hierarchical model. The variance at the group level constituted $4.3 \%$ and $1.3 \%$ of the total variance, respectively.

EVALUATION OF THE GROUP DISCUSSION PROCESS The mean (SD) scores of group discussion were $5.24(0.44)$ and $5.18(0.45)$ in the asthma $(n=90)$ and UTI $(n=86)$ groups, respectively. The mean prescription feedback scores were $5.39(0.69), n=82$, and $5.14(0.78), n=80$. Of 70 respondents in the asthma groups, $73 \%$ indicated that they would, $23 \%$ that they probably would, and $4 \%$ that they would not change their treatment of asthma as a result of the intervention, and of the 72 respondents in the UTI groups $89 \%$ indicated that they would, $6 \%$ that they probably would, and $6 \%$ that they would not change their treatment of UTI. Eighty (93\%) in the asthma groups and 77 $(96 \%)$ in the UTI groups judged the educational intervention used to be suitable also for changing treatment of other diseases.

\section{VARIATION IN THE CHANGE OF PRESCRIBING} BEHAVIOUR AND CHARACTERISTICS OF THE DOCTORS

The age, sex, number of workdays per week, years of experience, or board certification of GPs (table 3) did not significantly affect the change in prescribing behaviour.

Variation in the increase of acceptably treated asthma patients among all doctors was partly explained $\left(R^{2}=0.258\right)$ by the interven- 


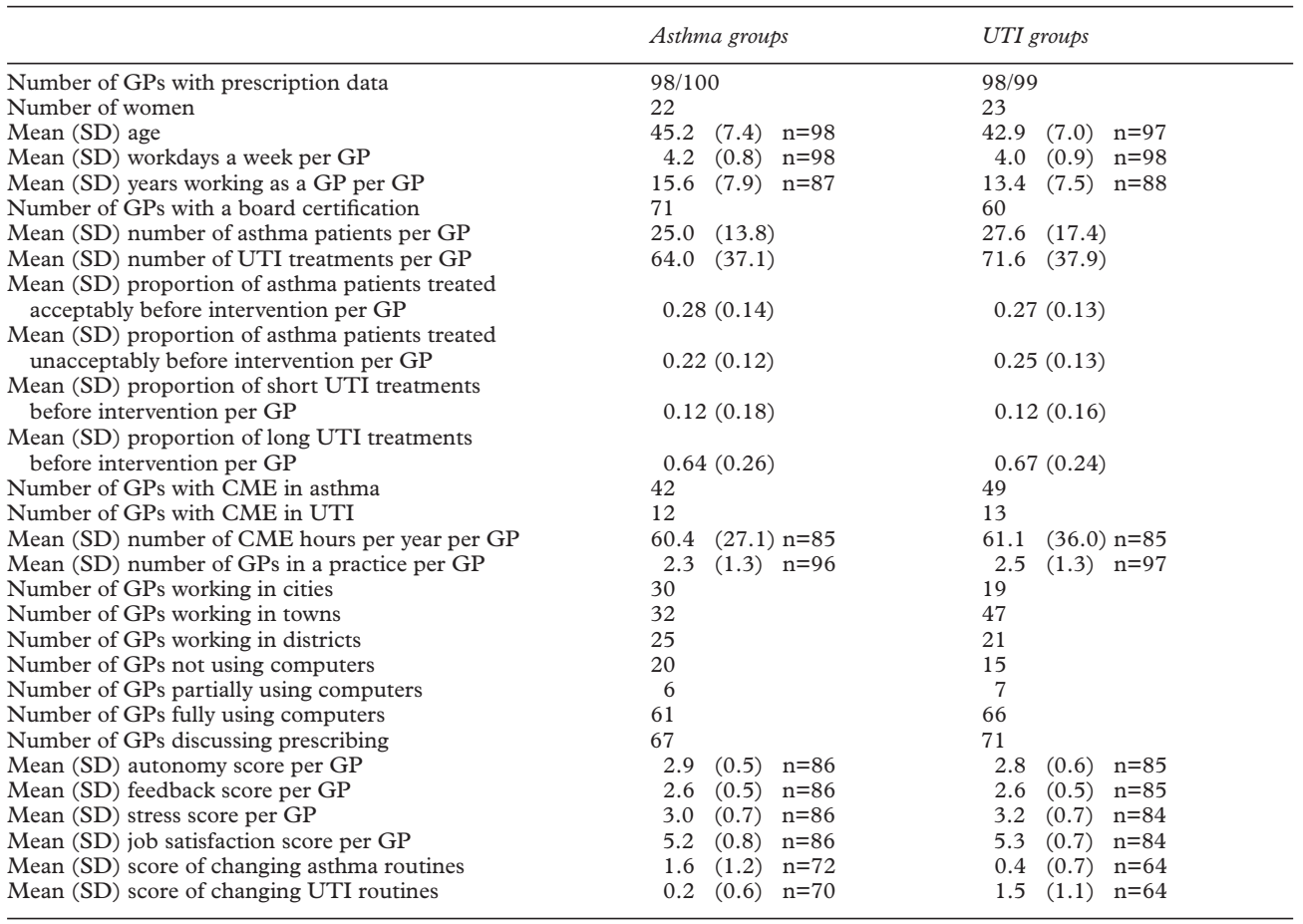

tion group to which they were allocated $(\beta=$ $-0.300, p<0.0004)$, participation in CME on asthma during the previous two years $(\beta=$ $0.253, \mathrm{p}=0.002)$, and the use of computers coded $1-3$ with increased use $(\beta=0.235, \mathrm{p}=$ $0.008)$. In general the $\beta$ coefficient is interpreted as the expected change in response corresponding to an increase of one unit. In this case the expected difference between the asthma group coded as 1 and the UTI group coded as 2 is $\beta=-0.300$. It was affected inversely by the job satisfaction score coded $1-10$ with increased satisfaction $(\beta=-0.202, p$ $=0.015)$ and by the proportion of acceptably treated patients before the intervention $(\beta=$ $-0.181, \mathrm{p}=0.030$ ).

Variation in the reduction of unacceptably treated asthma patients could, to some extent, be explained $\left(R^{2}=0.089\right)$ by stated improvement in management routines coded $0-3(\beta=$ $0.233, p=0.009)$ and participation in CME on asthma during the previous two years $(\beta=$ $0.177, \mathrm{p}=0.047$ ).

Variation in the increase of acceptable treatments for UTI among all doctors is partly explained $\left(R^{2}=0.313\right)$ by the intervention group to which they were allocated $(\beta=0.243$, $\mathrm{p}=0.013)$ and stated improvement of management routines $(\beta=0.282, \mathrm{p}=0.005)$. It was affected inversely by a practice located in an area with many rather than few inhabitants (city/town/district) $(\beta=-0.207, \mathrm{p}=0.010)$.

Variation in the reduction of unacceptable treatments for UTI is partly explained $\left(R^{2}=\right.$ 0.199 ) by which of the two intervention groups the doctors were allocated to $(\beta=0.266, \mathrm{p}=$ 0.002 ) and by the autonomy score of the GP coded 1-6 with increasing autonomy $(\beta=$ $0.201, p=0.027)$. It was affected inversely by a practice located in an area with many rather than few inhabitants (town/city/district) $(\beta=$
$-0.226, p=0.009)$ and by the number of GPs working together at the health care centre $(\beta=$ $-0.183, p=0.037)$.

\section{Discussion}

The educational intervention used in this study improved the prescribing behaviour of doctors in accordance with guideline recommendations, and is thus an aid in significantly improving the quality of patient care. Similar interventions using comparison of behaviour with recommended behaviour have previously been shown to improve requests by GPs for laboratory tests. ${ }^{24}$

In recording doctors' behaviour the drugs dispensed rather than those prescribed were used. The agreement between these two ways of recording drug use may be less than desired, reflecting the difference in behaviour between the patient and the doctor. ${ }^{25}$ The outcome measurement of dispensed drugs also includes the effect of the GP as a teacher and motivator, and relates somewhat more to the quality of patient care than recording only what the doctor prescribed.

A behavioural change resulting in a doctor doing more things "correctly" usually implies an inverse relationship of doing fewer things "wrong". In our study the linkage between an increase in acceptable treatment of asthma and a reduction in unacceptable treatment was not obvious since the proportion of patients difficult to classify was high. Most of those who were difficult to classify were using inhaled $\beta$ agonists in low mean daily dose as single medication. The threshold use of inhaled $\beta$ agonists before introducing inhaled steroids has changed during the last few years with a lower level being recommended in the most recent guidelines compared with earlier versions. ${ }^{14}$ Some, but not all, of the patients who were dif- 
ficult to classify should probably have been offered inhaled steroids. Only one fifth of the UTI treatments remained unclassified, which made the shift from unacceptable to acceptable treatments visible (table 3 ).

This study detected a significant change in behaviour despite extrinsic factors that could mask these observations. Promotional activities by the pharmaceutical industry using the asthma guidelines can be assumed to have influenced the prescribing behaviour of GPs in a desired direction in both the asthma and UTI groups. These groups were located geographically close to each other in order to minimise differences in external influences. This, however, increased the possibility of a leakage of factors influencing behaviour between the groups. Participation of the pharmacists in the groups may also have had an effect in diluting the differences as they saw patients from both study groups. The intervention was not expected to affect all antibiotics used as a proxy for UTI treatments. Co-trimoxazole, for instance, is also to some extent used for treating respiratory infections over a longer duration.

The group discussion and the feedback on prescribing was well regarded by the participants, and the intervention was judged suitable for improving the quality of treatment of other diseases such as hypertension and diabetes. A high proportion of the participants in our study stated they would change their treatment after the intervention. Self-rating has previously been shown to relate to an actual change in behaviour. ${ }^{26}$ We thus have reason to believe that the change demonstrated in our study was not limited to small subgroups of participants. It might therefore be worth using this intervention as a model for developing a CME programme since it has shown its ability to change behaviour. Being board certified was more common among the participating than among the non-participating GPs (table 2). These specialist GPs may be more accustomed to working in peer groups than their colleagues as peer reviewing is part of the training to become board certified in Norway. The educational programme used as an intervention may thus best accommodate the learning styles of GPs who enjoy group discussions.

The variance in behavioural change between the participating groups was less than $10 \%$ of the total variance, indicating that the change in prescribing behaviour was primarily related to individual characteristics of the GPs and their practice. Furthermore, the intervention process itself appears to have affected the groups equally as the process evaluations of the groups were uniform.

On the individual level, previous participation in CME on asthma was linked to improved behaviour; this may be because these GPs were more interested and thus more motivated to improve their asthma treatment than other GPs. The GPs who used computers in their work may indicate a group of doctors who more readily accept and use new techniques and therapies. The use of computers might also make it easier to trace patients in need of better treatment. ${ }^{27}$
Changing management routines is important in changing clinical practice. ${ }^{11}$ In our study this was the only factor, apart from previous participation in CME activity, that affected a change in the proportion of unacceptably treated asthma patients. Better routines may improve the relationship with these patients, making it possible to give better treatment. Nurses or other team members at the health centre in Norway often handle the management of acute or recurrent cystitis. Improved routines may thus ensure that all health workers in a team are uniformly informed about treatment recommendations.

A difficult job situation may be a barrier to improving the quality of patient care. ${ }^{28}$ In our study the doctors' experience of autonomy was favourably related to improving treatment of patients with UTI seeking immediate care. An overall favourable judgement of the working conditions, however, seemed to impede improvements in the care of asthma patients. One possible explanation for this may be that these GPs conform better to their working environment and thus are less motivated to change.

\section{Conclusion}

Combining prescription feedback of individual treatments with quality criteria of prescriptions based on guideline recommendations in peer review groups of doctors improved the quality of care of patients with asthma and urinary tract infection. This educational activity was valued by the doctors and judged suitable for improving the quality of care in other treatment areas. Some characteristics of the doctors and their practice of importance to their improvement were identified. Awareness of these factors may be important when discussing how to improve treatments and designing programmes for continuing medical education.

The project was supported financially by The Norwegian Medical Association's Fund for Quality Improvement, The Research Council of Norway, and The Norwegian Community Pharmacy Foundation. The authors thank Leiv Sandvik, Foundation for Health Services Research, for help with the multilevel calculations. The other members of the European Drug Education Project who made important contributions to the study are: F M Haaijer-Ruskamp (international co-ordinator), P Denig, and C C M Veninga (The Netherlands); V Diwan, G Tomson, R Wahlström, T Oke, and C Stålsby Lundborg (Sweden); M M Kochen and E Hummers-Pradier (Germany); M Muskova and ing general practitioners and pharmacies in Norway.

1 Grol R. Implementing guidelines in general practice care. Quality Health Care 1992;1:184-91.

2 Bero LA, Grilli R, Grimshaw JM, et al, on behalf of the Cochrane Effective Practice and Organisation of Care Review Group. Getting research findings into practice. Closing the gap between research and practice: an overview of systematic reviews of interventions to promote the implementation of research findings. BMF 1998;317:4658.

3 Hogerziel HV. Promoting rational prescribing: an international perspective. Br f Clin Pharmacol 1995;39:1-6.

4 Grimshaw JM, Russell IT. Achieving health gain through clinical guidelines II: Ensuring guidelines change medical practice. Quality Health Care 1994;3:45-52. 5 Gifford DR, Holloway RG, Frankel MR, et al. Improving opinion leaders. Ann Intern Med 1999;131:237-46.

6 Ullman LP, Krasner L. A psychological approach to abnormal behavior. Cap 12: behavior modification. New Jersey: Prentice-Hall, 1975: 224-47.

7 O'Connell DL, Henry D, Tomlins R. Randomised controlled trial of effect of feedback on general practitioners' preled trial of effect of feedback on general practition
scribing in Australia. BMF 1999;318:507-11.

8 Kanouse D, Itzihak J. When does information change prac-

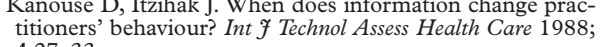
4:27-33. 
9 Spencer JA, Jordan RK. Learner centred approaches in medical education. BMF 1999;318:1280-3.

10 Bandura A. Self-efficacy. The exercise of control. New York: WH Freeman, 1997: 3.

11 Allery LA, Owen PA, Robling MR. Why general practitioners and consultants change their clinical practice: a critical incident study. BMF 1997;314:870-4.

12 Coles C, Holm HA. Learning in medicine: towards a theory of medical education. Oslo: Scandinavian University Press, 1993: 189-209.

13 Denig P, Andrew M, Wahlström R, et al. Results from an educational intervention in five countries to implement guidelines for prescribing in practice. Pharmacoepidemiology Drug Safety 1998;7:S177.

14 Vennerød AM, ed. Norwegian drug and therapeutic formulary. Oslo: Norsk legemiddelhåndbok I/S, 1994.

15 British Thoracic Society, British Paediatric Association, Royal College of Physicians of London, et al. Guidelines on management of asthma. Thorax 1993;48(Suppl 1):S1-24.

16 Stamm WE, Hooton TM. Management of urinary tract infection in adults. N Engl f Med 1993;329:1328-34.

17 Guidelines for ATC classification and DDD assignment. Oslo: WHO Collaboration Centre for Drug Statistics MethodWHO Colla

18 Aasland OG, Olff M, Falkum E, et al. Health complaints and job stress in Norwegian physicians: the use of an overlapping questionnaire design. Soc Sci Med 1997;45:161529.
19 Goldstein H, ed. Multilevel statistical models. London: Arnold, 1995

20 Rice R, Leyland A. Multilevel models: applications to health data. F Health Serv Res Policy 1996;1:154-64.

21 Goldstein H, Rasbash J, Plewis I, et al. A user's guide to MlwiN, version 1.0. Multilevel Model Project, Institute of Education, University of London, 1998.

22 SPSS. Statistical Package for Social Sciences, Version 7.5. Chicago: SPSS Inc, 1997.

23 Larsen Ø, ed. Doctors in Norway 1996. Oslo: The Norwegian Medical Association, 1996.

24 Winkens RAG, Pop P, Bugter-Maessen AMA, et al. Randomised controlled trial of routine individual feedback to improve rationality and reduce number of test requests. Lancet 1995;345:498-502.

25 Nilsson J L, Johansson H, Wennberg M. Large differences between prescribed and dispensed medicines could indicate undertreatment. Drug Information $\mathcal{f}$ 1995;25:1243-6.

26 Curry L, Purkins IE. Validity of self-reports on behaviour changes by participants after a CME course. $7 \mathrm{Med}$ Educ 1986;61:579-84.

27 Elson RB, Connelly DP. Computerized patient records in primary care. Their role in mediating guideline-driven physician behavior change. Arch Fam Med 1995;4:698-

28 Grol R, Mokkink H, Smith A, et al. Work satisfaction of general practitioners and the quality of patient care. Fam Practice 1985;2:128-35.

\section{È technè macrè (Art is long)}

Hippocrates

It happened.

She knew it happened,

Yet Tom was walking again, on the beach, with his grandchild.

Tom was grateful his young doctor caught the nasty bug early, while still in the hospital.

"It will happen," she was told, "a question of time, probability and bad physiology."

She knew. Yet, if art reflects accumulated experience,

How much art does she need to learn about her science?

"Outcomes count," an older colleague told her.

"Outcomes are what people see.

But your real art is to foresee the processes, to shape the pathways,

For they are personalized and yet generic. You are part of a system, every captain is."

From her window, she could see the beach, where waves come and waves disperse upon the sand.

On her laptop's screen, she has the day's headlines on medical errors and social accountability.

Yet in her mind, accountability seemed to still search for its audience, carefully.
She caught the bug early, alright, but that bug should have not infected Tom, in the first place.

Her colleagues welcomed her to the reality of the cline of which Tom was so proud.

Perhaps because Tom was untold of what to expect.

Yet his young doctor was unaware of how often such bugs find their Toms, elsewhere.

"If I know, I can educate my patients better," she thought,

Since expectations best predict satisfaction.

And she looked at the new waves crushing upon the beach

And the beach where Tom takes his grandchild in search of a silver dollar shell,

And she realized, that before telling Tom, she should know herself.

Until then, no index finger

Aimed at her face

Seemed appropriate.

VAHÉ A KAZANDJIAN 